\title{
ELETROCARDIOGRAFIA DE CÃES SUBMETIDOS A DIFERENTES CONCENTRAÇÕES DE DESFLURANO, PRÉ-TRATADOS OU NÃO COM A ASSOCIAÇÃO DE FENTANIL/DROPERIDOL
}

\begin{abstract}
ELECTROCARDIOGRAPHY OF DOGS UNDERGOING DIFFERENT DESFLURANE CONCENTRATIONS, PREMEDICATED OR NOT WITH FENTANYL/DROPERIDOL ASSOCIATION
\end{abstract}

\author{
Paulo Sérgio Patto dos Santos ${ }^{1}$ Newton Nunes ${ }^{2}$ Felipe Antônio Mendes Vicenti ${ }^{1}$ \\ Silvio Emílio Cuevas Martins ${ }^{3}$ Márlis Langenegger de Rezende ${ }^{1}$
}

\section{RESUMO}

\begin{abstract}
Avaliaram-se os efeitos da associação de fentanil e droperidol sobre a freqüencia cardíaca $(F C)$ e eletrocardiografia (ECG), em cães submetidos à anestesia pelo desflurano. Para tal, foram empregados quarenta animais hígidos, adultos, machos e fêmeas, com peso variando entre 6,5 e $20 \mathrm{~kg}$. Os cães foram separados igualmente em dois grupos G1 e G2. Ao G1, administrou-se fentanil $(15,7 \mu \mathrm{g} / \mathrm{kg})$ associado ao droperidol $(0,5 \mathrm{mg} / \mathrm{kg})$; ao $\mathrm{G} 2$, administrou-se solução fisiológica a $0,9 \%$ (placebo), ambos por via intravenosa. Decorridos 15 minutos, induziu-se à anestesia geral com administração intravenosa de propofol $(5 \mathrm{mg} / \mathrm{kg})$. Ato contínuo, os cães foram submetidos a anestesia inalatória pelo desflurano, diluído em oxigênio, através de circuito anestésico semifechado na concentração inicial de 1,6 CAM. As observações das variáveis tiveram início imediatamente antes da aplicação dos fármacos M1. Novas mensurações foram realizadas 15 minutos após a administração da associação fentanil/droperidol ou placebo M2 e 20 minutos decorridos da indução anestésica M3 (1,6 CAM). As demais colheitas coincidiram com o estabelecimento das concentrações de 1,4; 1,2; e 1,0 CAM, respectivamente M4 a M6. Os dados numéricos destas variáveis foram submetidos à Análise de Perfil, sendo considerado o nivel de significância de $p<0,05$. Os resultados permitiram concluir que a associação de fentanil/droperidol reduz a freqüencia cardíaca e o desflurano bloqueia a bradicardia produzida pela associação e promove retardo na condução elétrica atrial e ventricular.
\end{abstract}

Palavras-chave: eletrocardiografia, CAM, desflurano, fentanil, droperidol, cães.

\begin{abstract}
SUMMARY
The aim of this work was to evaluate the effects of the fentanil/droperidol association over the heart rate (HR) and electrocardiography (ECG) of dogs submitted to anesthesia by desflurane. For that, forty healthy adult dogs, males and females were used. The dogs were equally separate in two groups G1 and G2. G1 received fentanil $(15.7 \mu \mathrm{g} / \mathrm{kg})$ associated to droperidol $(0.5 \mathrm{mg} / \mathrm{kg}) ;$ while $\mathrm{G} 2$ received saline solution at $0.9 \%$ (placebo), both applied intravenously. After 15 minutes the general anesthesia was induced with intravenously administration of propofol $(5 \mathrm{mg} / \mathrm{kg})$. The dogs were submitted to inhalatory anesthesia with desflurane, with an initial concentration of 1.6 $M A C$. The observations of the variables were begun immediately before the application of the agents (M1). New measurings were carried out 15 minutes after the administration of the fentanil/droperidol association or placebo (M2) and 20 minutes after the anesthetic induction (M3). The other measurings coincided with the establishment of the concentrations of $1.4 ; 1.2$ and 1.0 MAC, respectively M4 to M6. The numeric data were submitted to the Analysis of Profile, being considered the level of significance of $p<0.05$. The results allowed to conclude that the fentanil/droperidol association decreases HR and the desflurane blocks the decrease on HR produced by the association and promoves a delay in the electrical atrial and ventricular conduction.
\end{abstract}

Key words: electrocardiography, MAC, desflurane, fentanyl, droperidol, dogs.

\footnotetext{
${ }^{1}$ Pós-graduando do Curso de Doutorado em Cirurgia Veterinária - Área de Concentração em Cirurgia - Faculdade de Ciências Agrárias e Veterinárias (FCAV), Universidade Estadual Paulista (UNESP), Campus de Jaboticabal, SP.

${ }^{2}$ Professor Assistente, Doutor, Departamento de Clínica e Cirurgia Veterinária. FCAV/UNESP, 14870-000, Jaboticabal, SP. E-mail: newton@fcav.unesp.br. Autor para correspondência.

${ }^{3}$ Pós-graduando do Curso de Mestrado em Cirurgia Veterinária - Área de Concentração em Cirurgia - FCAV/UNESP, Campus de Jaboticabal, SP.
} 


\section{INTRODUÇÃO}

Concomitantemente aos avanços técnicocientíficos, a Anestesiologia Veterinária vem sendo munida de técnicas e fármacos, dotados de diferentes características, desenvolvidas de modo a tornar $\mathrm{o}$ ato anestésico seguro e adequado aos diferentes procedimentos cirúrgicos. Neste sentido, têm-se empregado fármacos capazes de proporcionar anestesia de indução rápida, manutenção isenta de riscos, além de analgesia residual e mínima taxa de biotransformação.

Dentre os anestésicos voláteis possuidores dessas características, o desflurano vem sendo utilizado com freqüência, principalmente devido ao seu reduzido coeficiente de solubilidade no sangue, embora seu efeito anestésico só seja obtido em altas concentrações, ao redor de $7,2 \mathrm{~V} \%$ em cães (CLARKE et al., 1996) e 6 a $7 \mathrm{~V} \%$ no homem (EGER, 1992).

A fim de que se proceda a anestesia com a menor quantidade possível de anestésico e, conseqüentemente, com reduzido fator de risco, procura-se empregar agentes pré-anestésicos dotados de características que possam diminuir a concentração alveolar mínima (CAM) do agente volátil. Assim sendo, o pré-tratamento dos pacientes a serem anestesiados pelo desflurano assume importância, visto que se poderia abdicar de elevadas concentrações do fármaco. Nesse sentido, a associação de fentanil e droperidol, por proporcionar efeito neuroléptico, acrescido de ação analgésica, poderia se constituir em pré-medicação adequada. Por outro lado, são escassas as informações relativas ao emprego da associação de fentanil/droperidol e desflurano em cães e as ações desses fármacos sobre variáveis fisiológicas. Entre estas, destaca-se a eletrocardiografia, visto tratar-se, atualmente, de método de mensuração básica da condição dos mecanismos responsáveis pela correta despolarização elétrica do coração, realizada rotineiramente nos períodos pré, trans e pósanestésicos.

O fentanil, derivado sintético da morfina é classificado como opióide agonista, apresentando elevada potência e efeitos analgésico e hipnótico intensos; apresenta rápida ação, ao ser administrado por via intravenosa ou intramuscular (THURMON et al., 1996). Sobre a atividade cardiovascular, o fentanil produz redução da freqüência cardíaca, além de uma variedade de arritimias devido a aumento da atividade vagal, como bloqueio atrioventricular de $2^{\circ}$ grau e contrações ventriculares e supraventriculares característicos de ritmo de escape (HENDRIX et al., 1995). Contudo, a administração prévia de sulfato de atropina, por via subcutânea, na dose de
$0,044 \mathrm{mg} / \mathrm{kg}$, pode evitar estes efeitos (MASSONE, 1994).

O droperidol, butirofenona dotada da capacidade de causar depressão do sistema nervoso central, age seletivamente, bloqueando as ações centrais da dopamina e da noradrenalina, na formação reticular mesencefálica (MASSONE, 1994). No sistema extrapiramidal, atua simulando a ação do ácido gama-aminobutírico (BOOTH \& McDONALD, 1992). No sistema cardiovascular, possui efeito bloqueador dos receptores do tipo $\alpha 1$ adrenérgico e, quando usado por via intravenosa, produz queda da pressão arterial e do volume sistólico (MASSONE, 1994). Na dose de $0,5 \mathrm{mg} / \mathrm{kg}$, esse fármaco exerce pouco efeito sobre o débito cardíaco, contudo reduz a resistência periférica total e a freqüência cardíaca (BOOTH \& McDONALD, 1992).

A associação de fentanil e droperidol, através do seu mecanismo de ação, nas áreas subcorticais do cérebro, produz analgesia intensa e sedação, embora não altere as funções corticais. Em cães pode produzir hipotensão por bloqueio dos receptores $\alpha_{1}$-adrenérgico periféricos e bradicardia (THURMON et al., 1996). A associação de fentanil/droperidol não aumenta o consumo de oxigênio pelo miocárdio, embora o droperidol, quando administrado em altas doses, possa deprimir a função ventricular esquerda (OSTHEIMER $\boldsymbol{e t}$ al., 1975).

O desflurano é anestésico volátil halogenado fluorinado, cujo baixo coeficiente de solubilidade sangue/gás $(0,42)$, permite rápido aumento ou diminuição da concentração alveolar o que torna rápidas a indução $\mathrm{e}$ a recuperação do paciente (EGER, 1992). A indução da anestesia por máscara não é recomendada, devido ao fato de o desflurano apresentar ação irritante sobre as mucosas. Sua utilização em pacientes adultos e pediátricos tem sido associada à produção de tosse $\mathrm{e}$ laringoespasmo (TINKER, 1992).

A administração do desflurano foi correlacionada ao aumento da atividade simpática, a qual atinge o pico máximo decorridos 5 minutos de exposição ao fármaco (PACENTINE et al., 1995). A ação simpatomimética deve-se a existência de sítios receptores nas vias aéreas superiores, que respondem rapidamente à elevação da concentração deste gás nos alvéolos. Essa resposta é resultante da ação irritante sobre a mucosa (MUZI et al., 1996b), porém, CIOFOLO \& REIZ (1999) constataram que o aumento da atividade simpática, em humanos, parece estar relacionada à ativação central e não à irritação das vias aéreas superiores. Relativamente à função cardiovascular, esse agente anestésico halogenado possui a propriedade de diminuir o 
débito cardíaco (PAGEL $\boldsymbol{e t}$ al., 1998), a pressão arterial e a resistência vascular sistêmica (WEISKOPF, 1995), embora promova aumento da freqüência cardíaca (CLARKE et al., 1996).

Quanto à sensibilização do músculo cardíaco, em pacientes submetidos a doses exógenas de adrenalina (MOORE $\boldsymbol{e t} \boldsymbol{a l}$., 1993), o fármaco possui propriedades inibitórias sobre arritmias ventriculares espontâneas, após infarto do miocárdio (NOVALIJA et $\boldsymbol{a l} .$, 1998). Por outro lado, inexistem na literatura, dados relativos a possíveis interferências da associação em teste, sobre variáveis eletrocardiográficas, notadamente aquelas relativas à duração e amplitude das ondas, bem como dos intervalos, entre estas, embora TILLEY (1992) tenha estabelecido os valores normais, destas variáveis, em cães despertos. Este experimento tem por objetivo avaliar efeitos eletrocardiográficos, em cães pré-tratados com a associação de fentanil/droperidol e anestesiados com diferentes concentrações de desflurano.

\section{MATERIAS E MÉTODOS}

Foram utilizados 40 cães adultos, machos e fêmeas, sem raça definida, com pesos variando entre 6,5 e $20 \mathrm{~kg}$, considerados sadios, evitando-se fêmeas prenhes ou em estro, fornecidos pelo canil do Hospital Veterinário "Governador Laudo Natel”, da Faculdade de Ciências Agrárias e Veterinárias FCAV/UNESP, Campus de Jaboticabal, SP.

Após serem selecionados aleatoriamente, os animais foram protocolados e separados em dois grupos de 20 (G1 e G2). Aos animais do G1, foram administrados, por via intravenosa, $15,7 \mu \mathrm{g} / \mathrm{kg}$ de fentanil associado a $0,5 \mathrm{mg} / \mathrm{kg}$ de droperidol, formando uma solução ${ }^{\text {a }}$. Decorridos 15 minutos, foi induzida a anestesia geral pela administração intravenosa de propofol ${ }^{\mathrm{b}}$, na dose média de $5 \mathrm{mg} / \mathrm{kg}$, para permitir a intubação orotraqueal.

Logo após, os cães foram intubados com sonda de Magill e, imediatamente, submetidos à anestesia inalatória pelo desflurano ${ }^{\mathrm{c}}$. $\mathrm{O}$ fármaco diluído em oxigênio puro foi administrado através de circuito anestésico tipo "semifechado", dotado de vaporizador $\mathrm{e}^{\mathrm{e}}$ termocompensado, microprocessado e calibrado para esse agente anestésico. O desflurano foi fornecido na concentração inicial de 1,6 CAM, considerando-se o parâmetro como sendo equivalente a $7,2 \mathrm{~V} \%$ (CLARKE et $\boldsymbol{a l}$., 1996), mensurada em equipamento digital $^{\mathrm{f}}$, cujo sensor foi adaptado na extremidade da sonda orotraqueal conectada ao circuito anestésico. Após 20 minutos do estabelecimento da concentração inicial, esvaziou-se o balão reservatório e a concentração foi reduzida em 0,2 CAM, sendo o circuito anestésico saturado com a nova mistura gasosa.
Aguardou-se a leitura da concentração expirada atingir aquela previamente determinada. Esse protocolo foi repetido em intervalos de 15 minutos até que fosse atingida a concentração anestésica equivalente a 1 CAM. Nos animais do G2, foi empregada a mesma metodologia, sendo a associação fentanil/droperidol substituída por solução fisiológica a $0,9 \%$ (placebo), administrada pela mesma via e em volume equivalente ao que seria utilizado da associação dos fármacos.

A freqüência cardíaca (FC) foi obtida nos diferentes tempos e para ambos os grupos, por eletrocardiógrafo. As observações tiveram início imediatamente antes da aplicação dos fármacos (M1) e novas mensurações foram realizadas 15 minutos após a administração da associação fentanil/droperidol ou placebo (M2) e 20 minutos decorridos do estabelecimento da concentração inicial do agente volátil (M3). As demais colheitas coincidiram com o estabelecimento das concentrações de 1,4; 1,2 e 1,0 CAM, respectivamente M4 a M6.

Para a avaliação do eletrocardiograma, foi empregado eletrocardiógrafo computadorizado e foram observados os valores referentes à duração e amplitude da onda $\mathrm{P}$, respectivamente Ps e PmV, intervalo entre as ondas P e R (PR), duração do complexo QRS (QRSs), amplitude da onda R $(\mathrm{RmV})$, duração do intervalo entre as ondas $\mathrm{Q}$ e $\mathrm{T}$ (QT) e intervalo entre duas ondas R (RR). O registro de eventuais figuras eletrocardiográficas anormais foi feito continuamente ao longo de todo o experimento, enquanto a colheita dos valores numéricos seguiu os tempos descritos para a FC. A avaliação estatística das variáveis foi efetuada por meio de Análise de Perfil (MORRISON, 1967 e CURI, 1980), considerando um nível de significância de $5 \%(\mathrm{p}<0,05)$.

\section{RESULTADOS}

A avaliação da freqüência cardíaca demonstrou que essa foi significativamente menor no G1 em relação ao G2 em M2, M3 e M4. A comparação dos momentos nos grupos separadamente revelou que, no G1, a média de M2 foi significativamente menor que as demais e, no G2, as médias apresentaram valores decrescentes a partir de M4.

Os valores referentes a Ps mostraram que os grupos não apresentaram diferenças estatísticas ao longo dos momentos. No estudo dos valores obtidos, observou-se que no G1 as médias de M1 e M2 foram menores que as demais e no G2, as médias de M1 e M2 foram menores que às de M3, M4 e M5. Já nas médias relativas a PmV, não foram observadas diferenças entre grupos. $\mathrm{O}$ estudo de cada grupo individualmente revelou que, no G1, as 
médias de M1, M3 e M6 foram maiores que a observada em M2 e a média de M3 foi superior à de M5. No G2, as médias de M4 e M5 foram menores que à de M3 e a M6 foi maior que a média de M4.

Quanto ao intervalo PR, na análise do grupo G1, observou-se que as médias de M1 e M5 foram menores que à de M2 e a média de M4 foi maior que à de M5. No G2, não foi constatada diferença significativa entre os momentos.

$\mathrm{O}$ estudo de QRS não apresentou variações significativas entre grupos. $\mathrm{O}$ estudo dos grupos isoladamente demonstrou que no G1 as médias de M4 a M6 foram significativamente maiores que à de M1 e a média de M4 foi superior à de M2. Já no G2, a média de M3 foi maior que às demais. Com relação à $\mathrm{RmV}$, nenhuma diferença significativa foi observada entre os grupos. $\mathrm{Na}$ avaliação individual, o G1 mostrou média de M2 maior que à de M1 e média de M6 superior à de M3, enquanto no G2 as médias de M5 e M6 foram maiores que às de M3 e M4; a média de M1 foi superior à de M3 e a de M6 maior que à média de M2. Relativamente ao intervalo QT, a comparação entre grupos revelou que em M2, M3, M5 e M6, o G1 apresentou valores médios maiores que os de G2. $\mathrm{O}$ estudo individual dos grupos revelou que, no G1, as médias apresentaram valores crescentes ao longo dos momentos. No G2, as médias de M1 e M2 foram menores que as de M3 a M6 e as médias de M3 e M4 foram inferiores às de M5.

$\mathrm{Na}$ análise de RR, observou-se que, em M2, o G1 apresentou média maior que à do G2, contudo, na observação isolada dos grupos, o G1 apresentou média de M2 superior às demais e, no G2, as médias de M3 a M5 foram menores que as de M1 e M6.

\section{DISCUSSÃO}

Os resultados, apresentados das diferentes variáveis estudadas permite tecer alguns comentários ou hipóteses. Neste sentido, a ação do desflurano no sistema cardiovascular, como fora citado por PACENTINE $\boldsymbol{e}$ t al. (1995), LEUNG \& PASTOR (1998) e CIOFOLO \& REIZ (1999), apresentou atividade simpatomimética, visto que as médias de freqüência cardíaca foram maiores com concentrações anestésicas elevadas do fármaco em G2. Esses achados estão de acordo com o efeito observado por (BRENET et al. 1998), embora não se tenha observado diferenças estatísticas da variável em relação aos pacientes despertos, como enunciado por CLARKE et al. (1996). Isso ocorreu provavelmente devido ao autor, em seu estudo, ter optado pela indução por máscara, potencializando assim o efeito simpático do agente. O G1 apresentou diminuição da freqüência cardíaca no momento da administração da associação de fentanil/droperidol (M2), fato este que corrobora os resultados obtidos por BOOTH \& MCDONALD (1992), HENDRIX et al. (1995) e THURMON et al. (1996), que afirmaram ser tal associação capaz de produzir bradicardia por aumento da atividade vagal. Em M3 notou-se aumento da freqüência cardíaca, a qual se manteve estável até M6, embora não tenha sido similar ao ocorrido no G2, provavelmente devido ao efeito da associação de fentanil/droperidol nos

Tabela 1 - Valores médios, seguidos dos desvios padrão ( $\pm \mathrm{s}$ ) das variáveis: freqüência cardíaca (FC), duração da onda $\mathrm{P}$ (Ps), amplitude da onda P (PmV), duração do intervalo P-R (PR), duração do complexo QRS (QRS), amplitude da onda R (RmV), duração do intervalo Q-T (QT) e intervalo entre duas ondas R (RR) obtidos em cães anestesiados com doses decrescentes de desflurano, prétratados com associação de fentanil/droperidol (G1) ou placebo $(\mathrm{G} 2)$.

\begin{tabular}{|c|c|c|c|c|c|c|c|}
\hline Variáveis & & M1 & M2 & M3 & M4 & M5 & M6 \\
\hline \multirow[t]{2}{*}{$\mathrm{FC}$} & G1 & $99,20 \pm 18,18^{\mathrm{a}}$ & $69,65 \pm 21,92^{b *}$ & $98,80 \pm 15,17^{\mathrm{a}^{*}}$ & $99,20 \pm 13,55^{\mathrm{a}^{*}}$ & $100,85 \pm 16,01^{\mathrm{a}}$ & $97,80 \pm 18,57^{\mathrm{a}}$ \\
\hline & G2 & $104,15 \pm 18,50^{\mathrm{abc}}$ & $106,95 \pm 20,01^{\text {abc** }}$ & $111,40 \pm 22,47^{\mathrm{ab} *}$ & $110,60 \pm 17,76^{\mathrm{a} *}$ & $106,45 \pm 17,86^{b}$ & $101,80 \pm 18,02^{\mathrm{c}}$ \\
\hline \multirow[t]{2}{*}{ Ps } & G1 & $54,60 \pm 10,73^{\mathrm{c}}$ & $55,60 \pm 9,68 \quad$ bc & $61,35 \pm 10,26^{\mathrm{a}}$ & $62,35 \pm 9,85^{\mathrm{a}}$ & $61,20 \pm 10,40^{\mathrm{a}}$ & $61,30 \pm 14,53^{a}$ \\
\hline & G2 & $49,50 \pm 9,06 \quad \mathrm{c}$ & $53,50 \pm 9,41 \quad$ bc & $60,65 \pm 7,71^{\mathrm{a}}$ & $58,75 \pm 9,81^{a}$ & $59,80 \pm 10,12^{\mathrm{a}}$ & $57,35 \pm 8,86 \quad$ ab \\
\hline \multirow[t]{2}{*}{$\mathrm{PmV}$} & G1 & $0,190 \pm 0,056^{\mathrm{ab}}$ & $0,158 \pm 0,048^{\mathrm{c}}$ & $0,203 \pm 0,053^{\mathrm{a}}$ & $0,192 \pm 0,057^{\mathrm{abc}}$ & $0,184 \pm 0,048^{\text {bc }}$ & $0,188 \pm 0,044^{a b}$ \\
\hline & G2 & $0,183 \pm 0,044^{\mathrm{abc}}$ & $0,192 \pm 0,074^{\mathrm{abc}}$ & $0,193 \pm 0,048^{\mathrm{a}}$ & $0,169 \pm 0,064^{\mathrm{c}}$ & $0,174 \pm 0,068$ bc & $0,189 \pm 0,059^{a b}$ \\
\hline \multirow[t]{2}{*}{ PR } & G1 & $100,80 \pm 13,13^{b c}$ & $109,50 \pm 15,08^{\mathrm{a}}$ & $103,15 \pm 16,09^{\mathrm{abc}}$ & $106,45 \pm 19,66^{\mathrm{ab}}$ & $99,65 \pm 18,54^{\mathrm{c}}$ & $103,90 \pm 25,51$ \\
\hline & G2 & $103,15 \pm 10,65^{\mathrm{a}}$ & $104,30 \pm 13,33^{\text {a }}$ & $108,55 \pm 16,85^{\mathrm{a}}$ & $105,10 \pm 19,74^{\mathrm{a}}$ & $107,35 \pm 22,57^{\mathrm{a}}$ & $107,85 \pm 19,18^{\mathrm{a}}$ \\
\hline \multirow[t]{2}{*}{ QRS } & G1 & $61,70 \pm 13,93^{\mathrm{c}}$ & $63,40 \pm 13,13$ bc & $65,70 \pm 10,25^{\mathrm{abc}}$ & $70,35 \pm 9,44^{\mathrm{a}}$ & $68,20 \pm 7,68^{\mathrm{ab}}$ & $66,70 \pm 9,25 \quad$ ab \\
\hline & G2 & $57,10 \pm 11,60 \quad$ f & $60,90 \pm 14,67 \quad$ ef & $78,50 \pm 42,61^{\mathrm{a}}$ & $65,00 \pm 10,63$ cde & $65,15 \pm 10,12^{\text {bcde }}$ & $63,05 \pm 8,03$ de \\
\hline \multirow[t]{2}{*}{$\mathrm{RmV}$} & G1 & $1,095 \pm 0,427^{\mathrm{cd}}$ & $1,170 \pm 0,49 \quad$ ab & $1,117 \pm 0,47 \quad b c$ & $1,132 \pm 0,47^{\mathrm{ac}}$ & $1,179 \pm 0,47^{\mathrm{abc}}$ & $1,186 \pm 0,45 \quad$ ad \\
\hline & G2 & $1,216 \pm 0,42$ acdef & $1,151 \pm 0,41 \quad$ bcef & $1,131 \pm 0,44^{\mathrm{b}}$ & $1,162 \pm 0,44$ bf & $1,230 \pm 0,45 \quad$ de & $1,249 \pm 0,47 \quad \mathrm{~d}$ \\
\hline \multirow[t]{2}{*}{ QT } & G1 & $213,45 \pm 19,84 \quad \mathrm{f}$ & $253,45 \pm 21,69$ & $269,05 \pm 29,05^{\text {cd* }}$ & $268,35 \pm 32,62 \quad \mathrm{~d}$ & $280,15 \pm 22,51 \quad b^{*}$ & $297,50 \pm 48,51^{\mathrm{a}^{*}}$ \\
\hline & G2 & $209,30 \pm 13,64$ e & $213,70 \pm 22,23$ & $245,00 \pm 24,86^{\mathrm{d} *}$ & $250,25 \pm 24,89^{\mathrm{cd}}$ & $258,55 \pm 25,99^{b *}$ & $264,40 \pm 36,38^{a b c *}$ \\
\hline \multirow[t]{2}{*}{$\mathrm{RR}$} & G1 & $687,35 \pm 160,57^{b c}$ & $922,40 \pm 288,87^{\mathrm{a} *}$ & $596,05 \pm 79,81 \quad \mathrm{f}$ & $611,50 \pm 95,36$ def & $607,90 \pm 112,52$ ef & $663,15 \pm 226,34^{\mathrm{cd}}$ \\
\hline & G2 & $647,10 \pm 195,79^{a}$ & $592,80 \pm 150,33^{\text {abcd* }}$ & $553,05 \pm 109,44^{\mathrm{d}}$ & $554,80 \pm 92,65^{\mathrm{cd}}$ & $566,00 \pm 90,10$ bcd & $619,20 \pm 114,25^{\mathrm{a}}$ \\
\hline
\end{tabular}

* Existe diferença estatística entre os grupos para este momento pela Análise de Perfil ( $\mathrm{p}<0,05)$.

Médias seguidas pelas mesmas letras, nas linhas, não diferem significativamente entre si pela Análise de Perfil ( $\mathrm{p}<0,05)$. 
demais momentos, o que produziu médias menores (PACENTINE et al., 1995).

Os valores obtidos do traçado eletrocardiográfico, no que se refere à duração da onda P (Ps), mostraram que o desflurano pode estar envolvido no retardo da condução elétrica atrial pois, em ambos os grupos, observou-se aumento das médias da duração da onda $\mathrm{P}$ assim que o desflurano foi administrado. Esse efeito comporta-se de modo dose-dependente pois, com a diminuição da concentração anestésica, observa-se também redução do Ps.

Embora este achado seja mais evidente no grupo controle (G2), pode-se atribuir ação da associação de fentanil/droperidol sobre a dinâmica elétrica cardíaca, nesse caso mantendo o retardo da condução elétrica mesmo em concentrações mais baixas. A explicação para o efeito observado não pode ser embasada no aumento do volume atrial, visto que, ao contrário do que se observa na administração da quetamina (NUNES et $\boldsymbol{a l} .$, 1997), com aumento da pré e pós-carga, o desflurano reduz, de maneira dose-dependente, a pressão arterial e o débito cardíaco (PAGEL et al., 1998; KARZAI $\boldsymbol{e t}$ al.,1999). Adicionalmente, o fentanil pouco interfere com o débito cardíaco (THURMON et al., 1996; ROSS et al., 1998), conseqüentemente os volumes circulantes tanto no sistema de resistência, como no de capacitância periféricos estariam aumentados (WEISKOPF, 1995). Os resultados relativos ao estudo da onda $\mathrm{P}$ poderiam ser melhor explicados, devido a uma possível ação dos agentes sobre os átrios, aumentando a impedância da musculatura cardíaca superior (SANTOS et al., 2000).

Os achados relacionados à amplitude da onda $\mathrm{P} \quad(\mathrm{PmV})$ não apresentaram diferenças significativas entre os grupos. No estudo dos grupos individualmente, apesar de serem encontradas diferenças estatísticas significativas com as diversas doses anestésicas empregadas, as médias obtidas mantiveram-se dentro da faixa considerada normal à espécie (TILLEY, 1992) logo, essas variações podem estar relacionadas a sensibilidade do teste estatístico e não à improváveis alterações volumétricas das cavidades atriais.

Com relação ao tempo de condução elétrica átrio-ventricular (PR), não houve diferenças significativas entre os grupos e, no G2, com as diversas doses de desflurano utilizadas. Contudo, no G1 observaram-se diferenças estatísticas significativas em alguns momentos, principalmente no ato da administração da associação de fentanil/droperidol, em que se constatou aumento no tempo de condução elétrica. Os valores encontrados, mesmo estando dentro da faixa de normalidade (TILLEY, 1992), podem ser conseqüência de mecanismo compensatório. A administração da associação de fentanil/droperidol produz diminuição da freqüência cardíaca e sendo o débito cardíaco (DC) produto da freqüência cardíaca (FC) pelo volume sistólico (VS); DC = FC x VS (MUIR \& MASON, 1996), a condução elétrica poderia se prolongar proporcionando maior tempo de enchimento ventricular e, conseqüentemente, maior volume sistólico, de modo que o débito cardíaco fosse mantido em valores normais.

Já os valores colhidos no estudo da duração do complexo QRS, mostraram não haver diferenças entre os grupos; entretanto, no grupo tratado com a associação de fentanil/droperidol (G1), observou-se discreto aumento no tempo de condução elétrica ventricular no início da administração do desflurano, contudo este aumento não foi crescente com a elevação da dose. No G2, a maior média foi obtida quando os animais foram submetidos à maior concentração anestésica (1,6 CAM), o que demonstra, a exemplo do que ocorreu com a onda $\mathrm{P}$, retardo na despolarização ventricular, com efeito mais evidente em concentrações maiores do agente volátil.

A redução na amplitude da onda $\mathrm{R}$ foi dose-dependente quanto à geração do estímulo elétrico responsável pelo evento mecânico da despolarização ventricular em ambos os grupos, sendo que esses não apresentaram diferenças significativas entre si, embora no G1, a maior média tenha ocorrido no momento da administração da associação de fentanil/droperidol. Alteração da impedância, provavelmente produzida pelo desflurano e pelo pré-tratamento com a associação de fentanil/droperidol, a exemplo do que fora observado com a musculatura atrial, com possível aumento da resistência elétrica, poderia justificar o fenômeno. É importante sugerir a criação ou adaptação de metodologia específica, para confirmar ou não estas assertivas.

O intervalo QT é utilizado para monitorar efeitos de fármacos e eletrólitos sobre a dinâmica cardíaca e está intimamente relacionado à freqüência cardíaca (OGUCHI \& HAMLIN, 1993). TILLEY (1992) sugere que a freqüência cardíaca e o intervalo QT são governados separadamente por neurônios simpáticos, que podem ou não serem ativados juntos. Tal afirmativa explicaria os valores crescentes do intervalo QT, em ambos os grupos, à medida em que as doses anestésicas são reduzidas, visto que o efeito simpatomimético proporcionado pelo desflurano (PACENTINE et al., 1995; CIOFOLO \& REIZ, 1999) é menor em baixas concentrações do anestésico. No G1, as médias do intervalo QT ao longo dos momentos foram maiores 
que as observadas em G2. Podem-se atribuir esses achados ao fato de o droperidol apresentar propriedades adrenolíticas (THURMON et $\boldsymbol{a l} .$, 1996) e o fentanil atividade parassimpatomimética (HENDRIX et al., 1995; PACENTINE et al., 1995). Esses efeitos, somados à ação das doses decrescentes do desflurano, podem ter sido responsáveis pelo achado. Concomitantemente, pode ter ocorrido efeito semelhante ao observado na musculatura atrial, em que se constatou retardo da condução pois, sob ação do fármaco, observou-se alteração na despolarização e repolarização dos ventrículos. Nesse caso, o feixe de Hiss e as fibras de Purkinje poderiam estar envolvidos, pois BLAIR et al. (1989) descreveram que o fentanil e o sufentanil prolongam a duração do potencial de ação das fibras de Purkinje, por ação direta na membrana.

$\mathrm{O}$ parâmetro RR que, em síntese, representa as alterações ocorridas na freqüência cardíaca (TILLEY, 1992), corresponde aos achados de CLARKE et al. (1996); MUZI et al. (1996a) e DANIEL et al. (1996), os quais, utilizando o desflurano, obtiveram aumento da freqüência cardíaca, que foi explicada pela ação simpatomimética do agente volátil (PACENTINE, et al., 1995; MUZI, et al., 1996b; EBERT, et al., 1995; EBERT, et al., 1998). O fato de as médias apresentarem valores crescentes, coincidentes com a redução da concentração de desflurano, fala a favor de tal hipótese, principalmente no grupo 1, no qual o efeito parassimpatomimético causado pelo fentanil (HESS, et al., 1989; HENDRIX, et al., 1995; DANIEL, et al., 1998) foi rapidamente suprimido quando se iniciou administração do desflurano.

\section{CONCLUSÕES}

Levando-se em conta os resultados obtidos, foi possível concluir que a associação de fentanil/droperidol reduz a freqüência cardíaca e a administração de desflurano bloqueia a bradicardia produzida pela associação, bem como promove retardo na condução elétrica atrial e ventricular.

\section{AGRADECIMENTOS}

Os autores agradecem à Fundação de Amparo à Pesquisa do Estado de São Paulo - FAPESP, pelo apoio financeiro concedido.

\section{FONTES DE AQUISIÇÃO}

${ }^{a}$ Nilperidol - Cristália Produtos Químicos e Farmacêuticos Ltda. ${ }^{b}$ Diprivan - ZENECA Farmacêutica do Brasil Ltda.

${ }^{\mathrm{c}}$ Suprane - ZENECA Farmacêutica do Brasil Ltda.

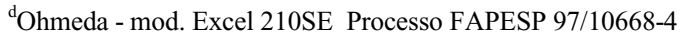

${ }^{\mathrm{e}}$ Ohmeda - mod. TEC 6 Processo FAPESP 97/10668-4

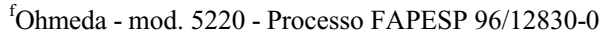

${ }^{\mathrm{g}}$ Teb - mod. ECGPC software versão 1.10 - Processo FAPESP 96/1151-5

\section{REFERÊNCIAS BIBLIOGRÁFICAS}

BLAIR, J.R., PRUETT, J.K., INTRONA, R.P., et al. Cardiac electrophysiologic effects of fentanyl and sufentanil in canine cardiac Purkinje fibers. Anesthesiology, v.71, n.4, p.565 570,1989

BOOTH, N.H., McDONALD, L.E. Farmacologia e terapêutica em veterinária. 6. ed. Rio de Janeiro : Guanabara Koogan, 1992. p.234 -267.

BRENET, O., GRANRY, J.C., POIRIER, N., et al. The effect of desflurane on cerebral blood flow velocity and cerebrovascular reactivity to $\mathrm{CO} 2$ in children. Ann Fr Anesth Reanim, v.17, n.3, p.227-233, 1998.

CIOFOLO, M.J., REIZ, S. Circulatory effects of volatile anesthetic agents. Minerva Anestesiol, v.65, n.5, p.232-238, 1999.

CLARKE, K.W., ALIBHAI, I.K., LEE, Y.H., et al. Cardiopulmonary effects of desflurane in the dog during spontaneous and artificial ventilation. Res Vet Sci, v.61, p.82-86, 1996.

CURI, P.R. Análise de medidas repetidas em experimentos biológicos. Rev Bras Estat, v.41, n.161, p.137-150, 1980.

DANIEL, M., EGER, E.I., WEISKOPF, R.B., et al. Propofol fails to attenuate the cardiovascular response to rapid increases in desflurane concentration. Anesthesiology, v.84, p.75-80, 1996.

DANIEL, M., WEISKOPF, R.B., EGER, E.I. Fentanyl augments the blockade of sympathetic response to incision (MACBAR) produced by desflurane and isoflurane. Anesthesiology, v.88, n.1, p.43-49, 1998.

EBERT, T.J., MUZI, M., LOPATKA, C.W. Neurocirculatory responses to sevoflurane in humans. A comparison to desflurane. Anesthesiology, v. 83, p.88-95, 1995.

EBERT, T.J., PEREZ, F., UHRICH, T.D., et al. Desfluranemediated sympathetic activation occurs in humans despite preventing hypotension and baroreceptor unloading. Anesthesiology, v.88, n.5, p.1227-1232, 1998.

EGER, E.I. Desflurane animal and human pharmacology: aspects of kinetics, safety, and MAC. Anesth Analg, v.75, p.3-9, 1992.

HENDRIX, P.K., ROBINSON, E.P., RAFFE, M.R. Methoctramine, a cardioselective muscarinic cholinergic antagonist, prevents fentanyl-induced bradycardia in the dog. J Vet Pharmacol Ther, v.18, n.2, p.87-93, 1995.

HESS, L., VRANA, M., VRANOVA, Z., et al. The electrostabilizing effect of a combination of midazolam and fentanyl: an experimental study in the dog. Cor Vasa, v.31, n.5, p.411-418, 1989.

KARZAI, W., HABERSTROH, J., PRIEBE, H.J. The efectts of increasing concentrations of desflurane on systemic 
oxygenation during one-lung ventilation in pigs. Anesth Analg, v.89, n.1, p.215-217, 1999.

LEUNG, J.M., PASTOR, D.A. Dissociation between haemodynamics and sympathetic activation during anaesthetic induction with desfluranes. Can J Anaesth, v.45, n.6, p.533-540, 1998.

MASSONE, F. Anestesiologia veterinária. Farmacologia e técnicas. 2.ed. Rio de Janeiro : Guanabara Koogan, 1994. $252 \mathrm{p}$.

MOORE, M.A., WEISKOPF, R.B., EGER, E.I., et al. Arrhythmogenic doses of epinephrine are similar during desflurane or isoflurane anesthesia in humans. Anestesiology, v.79, p.943-947, 1993.

MORRISON, D.F. Multivariate statistical methods. New York : MaC Grows Hill Book, 1967. 388p.

MUIR, W.W., MASON, D. Cardiovascular System. In: THURMON, J.C., TRANQUILLI, W.J., BENSON, G.J. Lumb \& Jones' veterinary anesthesia. 3. ed. Philadelphia: Lea \& Feabiger, 1996. p.80.

MUZI, M., LOPATKA, C.W., EBERT, T.J. Desflurane mediated neurocirculatory activation in humans. Effects of concentration and rate of change on responses. Anestesiology, v.84, p.1035-1042, 1996a.

MUZI, M., EBERT, T.J., HOPE, W.G., et al. Site(s) mediating sympathetic activation with desflurane. Anestesiology, v.85, n.4, p.737- 747, 1996 b.

NOVALIJA, E., HOGAN, Q.H., KULIER, A.H., et al Effects of desflurane, sevoflurane and halothane on postinfarction spontaneous dysrhythmias in dogs. Acta Anaesthesiol Scand,v.42, n.3, p.353-357,1998.

NUNES, N., CAMACHO, A.A., COSTA, J.L.O., et al. Eletrocardiographic study of the anesthetic combination of ketamine and chlorpromazine HCL in felines. Braz $\mathbf{J}$ Vet Res An Sci, v.34, n.1, p.313-316, 1997.
OSTHEIMER, G.W., SHANAHAN, E.A., GUYTON, R.A., et al. Effects of fentanyl and droperidol on canine left ventricular performance. Anesthesiology, v.42, p.288-291, 1975.

OGUCHI, Y., HAMLIN, R.L. Duration of QT interval in clinically normal dogs. Am J Vet Res, v.54, n.12, p. 21452149, 1993.

PACENTINE, G.G., MUZI, M., EBERT, T.J. Effects of fentanyl on sympathetic activation associated with the administration of desflurane. Anestesiology, v.82, p.823-831, 1995.

PAGEL, P.S., FU, J.L., DAMASK, M.C., et al. Desflurane and isoflurane produce similar alterations in systemic and pulmonary hemodynamic and arterial oxygenation in patient undergoing one-lung ventilation during thoracotomy. Anesth Analg, v.87, p.800-807, 1998.

ROSS, S., MUÑOZ, V., PIRIOU, V., et al. A comparison of the effects of fentanyl and propofol on left ventricular contractility during myocardial stunning. Acta Anaesthesiol Scand,v.42, p.23-31, 1998.

SANTOS, P.S.P., NUNES, N., VICENTI, F.A.M., et al. Estudo eletrocardiográfico, em cães tratados com concentrações decrescentes de desflurano. ARS Vet, v.16, n.1, p.16-21, 2000.

THURMON, J.C., TRANQUILLI, W.J., BENSON, G.J. Lumb \& Jones' veterinary anesthesia. 3. ed. Philadelphia : Lea \& Feabiger, 1996. Preanesthetics and anesthetic adjuncts: p.183-209.

TILLEY, L.P. Essentials of canine and feline electrocardiography: Interpretation and treatment. 3. ed. Philadelphia : Lea \& Feabiger, 1992. 470p.

TINKER, J.H. Desflurane: first new volatile anesthetic in almost 20 years. Anesth Analg, v.75, p.1-2, 1992.

WEISKOPF, R.B. Cardiovascular effects of desflurane in experimental animals and volunteers. Anaesthesia, v.50, p.14 -17, 1995. 